\title{
Measurement of Terahertz Optical-Beat Frequency Using High-Order Harmonics of Microwave in a Photoconductive Device
}

\author{
Kengo Murasawa, Koki Sato, and Takehiko Hidaka \\ Department of Applied Computer Sciences, Shonan Institute of Technology, 1-1-25 Tsujido-Nishikaigan, Fujisawa, \\ Kanagawa 251-8511, Japan \\ Correspondence should be addressed to Kengo Murasawa, sguest01@center.shonan-it.ac.jp
}

Received 31 May 2010; Accepted 22 March 2011

Academic Editor: Mikhail Noginov

Copyright ( $) 2011$ Kengo Murasawa et al. This is an open access article distributed under the Creative Commons Attribution License, which permits unrestricted use, distribution, and reproduction in any medium, provided the original work is properly cited.

A method for measuring frequencies of the terahertz $(\mathrm{THz})$ radiation emitted by the antenna mounted on the photoconductive (PC) device is presented. Two laser beams with slightly different frequencies irradiate the PC device, producing a beat current of $1 \mathrm{THz}$ in the photocurrent. A microwave signal is applied to the antenna electrode. The frequency of the THz wave is measured using the homodyne detection of the optical beat with the high-order harmonics of the microwave. It is being investigated that the high-order harmonics are produced by the PC device owing to its nonlinearity. Periodic peaks generated by the homodyne detection were observed in the photocurrent, as the microwave was swept from 16 to $20 \mathrm{GHz}$ with a power of $-40 \mathrm{dBm}$. Using the peak frequencies, the THz-wave frequency was determined to be $1030.3 \pm 3.73 \mathrm{GHz}$. The measurement error is estimated to be less than $0.43 \mathrm{GHz}$. The proposed method realizes a compact frequency meter in the $\mathrm{THz}$ region.

\section{Introduction}

A method for measuring frequencies of continuous wave $(\mathrm{cw})$ terahertz $(\mathrm{THz})$ radiation is one of key technologies for various applications of $\mathrm{THz}$ waves, for example, $\mathrm{THz}$ spectroscopy, radioastronomy, and future communication systems. Frequencies of the $\mathrm{THz}$ wave are usually measured using a Fourier-transformed interferometer which is applicable to both the $\mathrm{cw}$ and pulsed $\mathrm{THz}$ radiation. Various types of interferometers in the $\mathrm{THz}$ region are used, for example, a Martin-Puplett interferometer [1] and scanning FabryPerot etalon [2]. Frequency resolution of the interferometer is limited by the scanning range of the path difference of the $\mathrm{THz}$ wave. Longer scanning range is necessary to obtain higher frequency resolution. For example, the scanning range of 1 meter is necessary to obtain the frequency resolution of $0.15 \mathrm{GHz}$. Such an interferometer is generally large compared with the $\mathrm{THz}$ generation/detection system itself and is not suitable for practical use.

The $\mathrm{cw} \mathrm{THz}$ wave can be generated by a photomixing technique [3]. When two cw laser beams with slightly different optical frequencies $\left(f_{1}, f_{2}\right)$ irradiate the antenna gap fabricated on the photoconductive (PC) device, an opticalbeat current at a difference frequency $f_{\mathrm{T}}\left(=f_{1}-f_{2}\right)$ is produced between the electrodes (i.e., the antenna). The $\mathrm{THz}$ wave with the same frequency $f_{\mathrm{T}}$ is emitted from the antenna by dipole radiation. One method to know the frequency of the THz wave is by utilizing the locked difference frequency of the two laser beams of the light sources $[4,5]$. However, the setup required to lock the difference frequency of the two laser beams is apt to increase system complexity and system scale. Recently, a frequency of the pulsed $\mathrm{THz}$ wave was measured using a $\mathrm{THz}$-frequency comb induced by irradiating the PC device with the femtosecond laser 
pulses $[6,7]$. This method suggests that the homodyne or heterodyne detection techniques are useful for measuring the frequency of the $\mathrm{THz}$ wave.

In order to detect the $\mathrm{cw} \mathrm{THz}$-wave by the homodyne detection [8], two laser beams of the light sources are divided into two branches. One of the two branches irradiates the emitting PC device and emits the $\mathrm{THz}$ wave. The other branch irradiates the receiving PC device and causes the optical-beat current which simultaneously modulates the photoconductance of the PC device. In the receiving PC device, two signals with $\mathrm{THz}$ frequencies are multiplied. One of the two $\mathrm{THz}$ signals is the modulated photoconductance caused by the optical-beat current. The other is antenna voltage induced by the electric field of the received $\mathrm{THz}$ wave. Since the frequency of the modulated photoconductance is the same as that of the induced antenna voltage, the directcurrent $(\mathrm{dc})$ component is generated in the photocurrent by the homodyne detection.

If a voltage signal with a $\mathrm{THz}$ frequency $f_{\mathrm{T}}^{\prime}$ is, instead of the antenna voltage induced by the received $\mathrm{THz}$ wave, externally applied to the antenna of the receiving PC device, it is expected that the heterodyne signal (generally $f_{\mathrm{T}}^{\prime} \neq f_{\mathrm{T}}$ ) is generated by the similar principle mentioned above. When $f_{\mathrm{T}}^{\prime}=f_{\mathrm{T}}$, it is particularly expected that the dc component of the photocurrent is generated by the homodyne detection and can be detected by a lock-in amplifier. The voltage signal with the $\mathrm{THz}$ frequency can be generated by microwave harmonics using a suitable microwave diode. If such a diode generates high-order harmonics of the microwave with frequencies up to the $\mathrm{THz}$ region, a homodyne signal of the optical beat with the high-order harmonics of the microwave is expected.

In this paper, we report two subjects. First, the homodyne detection of the optical beat with the high-order harmonics of the microwave is described in Sections 2 and 3.1. In the experiments, it was found that the high-order harmonics of the microwave were generated by the PC device. Hence, the harmonic generation in the PC device is described in Section 3.2. Next, the method for measuring the frequency of the optical beat is described in Section 3.3. Procedures and results of the frequency measurement of the optical beat at about $1 \mathrm{THz}$ are explained and discussed.

\section{Experimental Setup}

Figure 1 shows the experimental setup to measure the optical-beat frequency in the PC device. A dual-wavelength distributed Bragg-reflecting laser diode (DW-DBR-LD) [9] was used as a light source. The DW-DBR-LD operates in cw and simultaneous dual-wavelength mode with a wavelength separation of approximately $2.48 \mathrm{~nm}(\approx 1 \mathrm{THz})$ centered at $850 \mathrm{~nm}$. The DW-DBR-LD can also oscillate in a singlewavelength mode by adjusting its injection current. The single-wavelength mode was used to compare the photocurrent response in the dual-wavelength mode with that in the single-wavelength mode. The output beam was routed to an optical isolator to avoid instability of the laser oscillation owing to backward reflection. The oscillation spectrum was monitored by an optical spectrometer (Hamamatsu
Photonics PMA-50, the wavelength resolution of $0.055 \mathrm{~nm}$ (24 GHz in frequency) at $850 \mathrm{~nm})$. The main beam passed through an attenuator (ATT) with which the incident laser power was controlled and was focused by an objective lens (L) onto the PC device. The PC material was a lowtemperature-grown GaAs (LTG-GaAs) film grown on a semi-insulating GaAs substrate [3] as shown in Figure 2. The dipole-antenna structure with a gap of approximately $5 \mu \mathrm{m}$ was located at the center of a $10-\mathrm{mm}$ long coplanar transmission line with a separation of approximately $30-\mu \mathrm{m}$. This antenna structure was fabricated on the LTG-GaAs film.

The PC device was biased to a voltage $V_{s}$ via a resistor $R_{s}(1 \mathrm{M} \Omega)$. The $\mathrm{THz}$ wave was emitted into free space. The microwave signal was supplied by a signal generator (SG) (Agilent $83624 \mathrm{~B}$, frequency range: $2-20 \mathrm{GHz}$ ) and applied to one of two antenna electrodes via a diode and capacitor (C). The other electrode was grounded. The diode was a step recovery diode (Herotek GC2050A, SMA type, efficiency of the harmonic generation $\approx-20 \mathrm{~dB} /$ octave). The diode was connected to an SMA-type connector assembled on the metal mount of the PC device. The capacitor $\mathrm{C}$ was a tip-type monolithic ceramic capacitor (Murata, capacitance of $10 \mathrm{pF}$ ). By modulating the amplitude of the microwave signal at $1 \mathrm{kHz}$, the resultant photocurrent variations were converted to the voltage variations by the resistor $R_{s}$ and detected by a lock-in amplifier (time constant: $1 \mathrm{~s}$ ).

Prior to the measurement of the optical-beat frequency, the THz-wave generation was confirmed by the homodynedetection technique using the setup shown in Figure 3. The $\mathrm{THz}$ wave emitted by the PC device was reflected by an aluminum-block concave mirror $(\mathrm{M})$ and focused onto the same PC device. That is, the PC device operates as a transceiver for the $\mathrm{THz}$ wave. The $\mathrm{THz}$ wave was chopped by a mechanical chopper $(13 \mathrm{~Hz})$. The resultant on-off homodyne current was converted to the on-off voltage by $R_{s}$ and measured by a lock-in amplifier.

The amplitude of the photocurrent variations was obtained while moving the mirror $\mathrm{M}$ along the emitting axis of the $\mathrm{THz}$ wave was measured. The maximum homodyne current of $1 \mathrm{nA}$ was obtained. The frequency of the THz wave was obtained by Fourier-transforming the data in which the mirror displacement was converted to the path difference of the $\mathrm{THz}$ wave in free space by multiplying by $2 / c$, where $c$ is the speed of light. The frequency of $1028 \mathrm{GHz}$ was obtained in the Fourier-transformed spectrum under the conditions of the incident laser power $P_{0}=4 \mathrm{~mW}$ and $V_{s}=20 \mathrm{~V}$. The frequency error was estimated to be $\pm 5 \mathrm{GHz}$, because the spectral width of the $\mathrm{THz}$ wave was narrower than the frequency resolution of $10 \mathrm{GHz}$ derived from the mirror displacement of $14 \mathrm{~mm}$. Therefore, the frequency of the optical beat is determined to be $1028 \pm 5 \mathrm{GHz}$, which is referred as the coarse frequency of the optical beat in Section 3.3.

\section{Results and Discussion}

3.1. Homodyne Detection of Optical Beat with Microwave Harmonics. The microwave signal was applied to the PC device via the diode as shown in Figure 1 and swept 


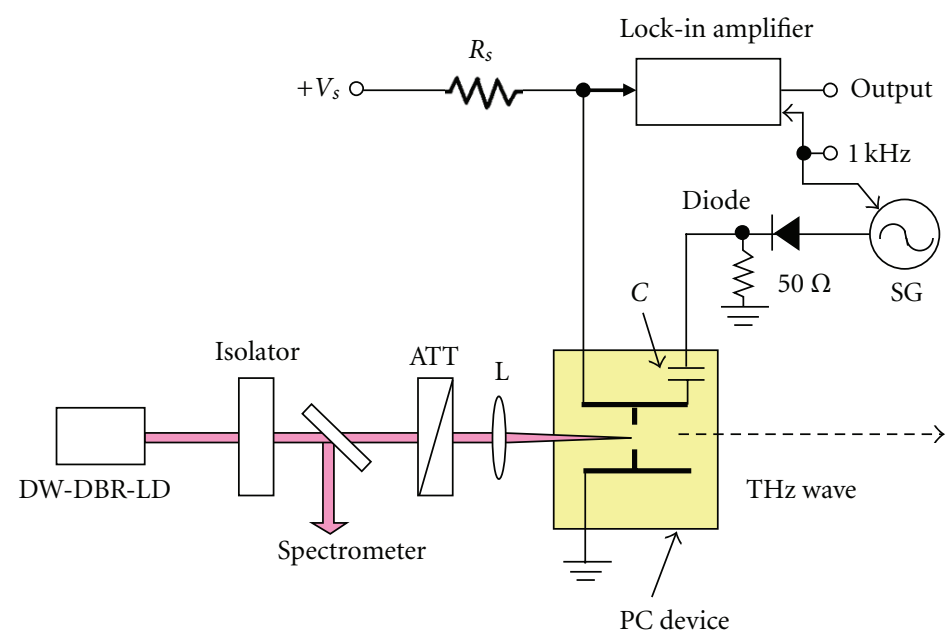

FIGURE 1: Experimental setup to measure the optical-beat frequency. By modulating the amplitude of the microwave signal at $1 \mathrm{kHz}$, the resultant photocurrent variations were detected by a lock-in amplifier.

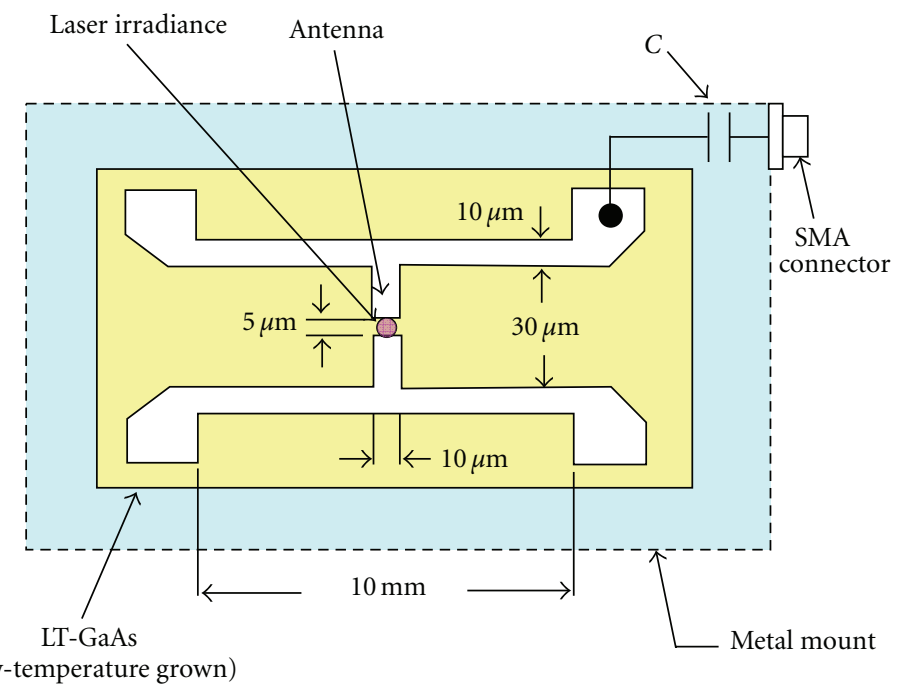

FIGURE 2: Structure of the PC device.

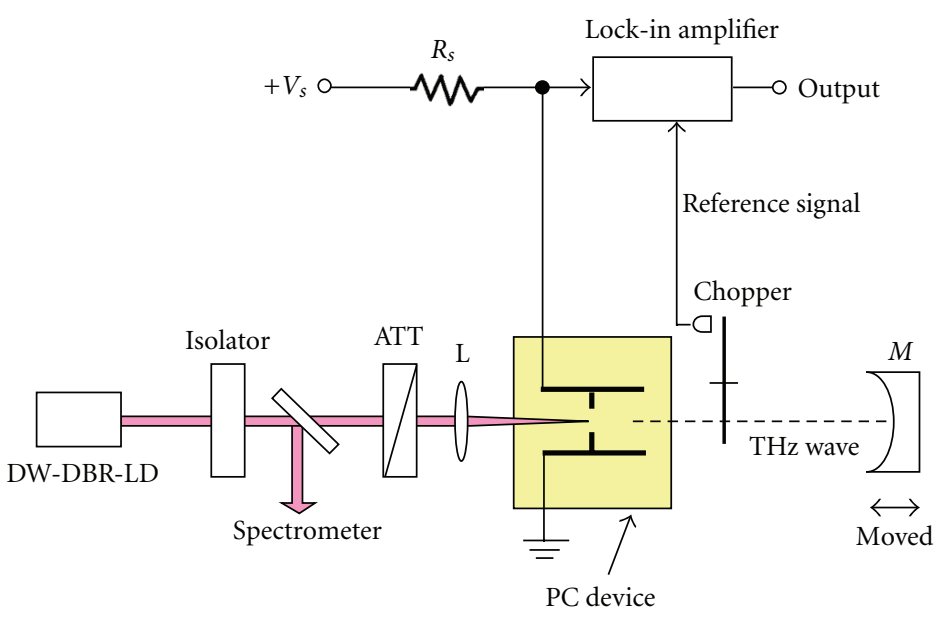

FigurE 3: Experimental setup of a THz-wave generator and homodyne receiver using a single PC device (THz transceiver). The emitted $\mathrm{THz}$ wave was reflected by a mirror $(\mathrm{M})$ and focused onto the same PC device. 
from 16 to $20 \mathrm{GHz}$ (sweep rate: $1 \mathrm{MHz} / \mathrm{s}$ ) with a power of $P_{\mathrm{mw}}=-40 \mathrm{dBm}$. Figure 4 shows the dependence of the photocurrent on the microwave frequency plotted every $1 \mathrm{MHz}$ in the microwave frequency. Figure 4(a) shows the photocurrent variation in the case of dual-wavelength irradiation of the laser light (i.e., optical beating at about $1 \mathrm{THz}$ ). Clear periodic peaks in the photocurrent were observed as the microwave frequency was swept. These periodic peaks were observed only in the microwave power range of $P_{\mathrm{mw}}=-43$ to $-37 \mathrm{dBm}$ (power variation: $\pm 3 \mathrm{~dB}$ ). Figure 4(b) shows the photocurrent variation in the case of single-wavelength irradiation of the laser light (i.e., no optical beating). No periodic peak was observed. Therefore, the periodic peaks shown in Figure 4(a) are definitely caused by the optical beat. Figure 4(c) show the photocurrent variation in the case of no-irradiating while biasing the PC device. Figure $4(\mathrm{~d})$ shows the photocurrent variation in the case of no-irradiating and no-biasing the PC device. No periodic peak was observed for both the cases. Therefore, it is obvious that the periodic responses shown in Figure 4(a) are not parasitic resonances of the electric circuits. Because the peaks shown in Figure 4(a) periodically occur as the microwave frequency is swept, these peaks are most likely generated by the homodyne detection of the optical beat with the high-order harmonic of the microwave when the frequency of the high-order harmonic of the microwave is tuned to that of the optical beat. Since the oscillation frequencies of the DW-DBR-LD are not stabilized, a difference between the two oscillation frequencies randomly fluctuates around an average of the difference frequencies. When the swept frequency of the high-order harmonic of the microwave coincides with the instantaneous frequency of the optical beat, the peak of the photocurrent is generated by the homodyne detection. Accordingly, the peak width in Figure 4(a) exhibits the frequency fluctuation of the optical beat caused by the fluctuation of the difference frequencies of the incident laser light.

3.2. Characteristics of Harmonic Generation in the PC Device. If the periodic peaks in Figure 4(a) are generated by the homodyne detection, the high-order harmonics with the $\mathrm{THz}$ frequencies must be generated by the diode and reach the antenna. However, since a length between the diode and antenna in our setup was approximately $20 \mathrm{~mm}$, the harmonics with frequencies higher than $100 \mathrm{GHz}$ are difficult to reach the antenna. Hence, it is expected that nonlinearities are caused in the PC device. In order to confirm whether the nonlinearity exists in the PC device or not, we measured the efficiency of the high-order harmonic generation $(\eta)$ using the setup shown in Figure 5. An electrically tunable dualwavelength laser diode (TDW-LD) [10] was used as a light source. The wavelength separation of the TDW-LD can be electrically tuned to $0-4.4 \mathrm{~nm}$ (approximately $0-2.2 \mathrm{THz}$ ) at the center wavelength of $780 \mathrm{~nm}$. Here, the wavelength separation of $0 \mathrm{~nm}$ means for the TDW-LD to oscillate in the single-wavelength mode. The wavelength separation of the TDW-LD was set to 2,4 , or $0 \mathrm{~nm}$ to generate the opticalbeat frequencies at $1 \mathrm{THz}, 2 \mathrm{THz}$, and no optical beating in the PC device, respectively. A low-frequency signal $(600 \mathrm{~Hz})$

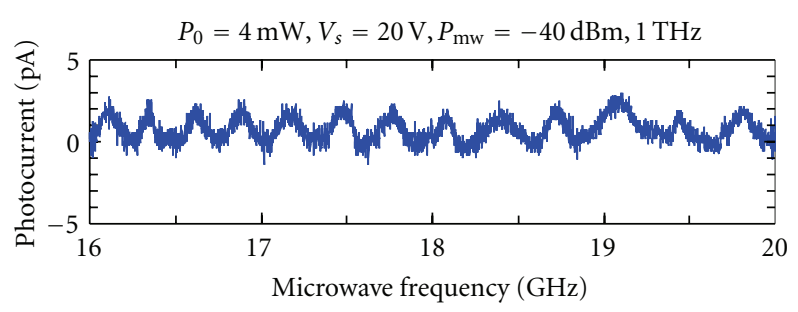

(a)

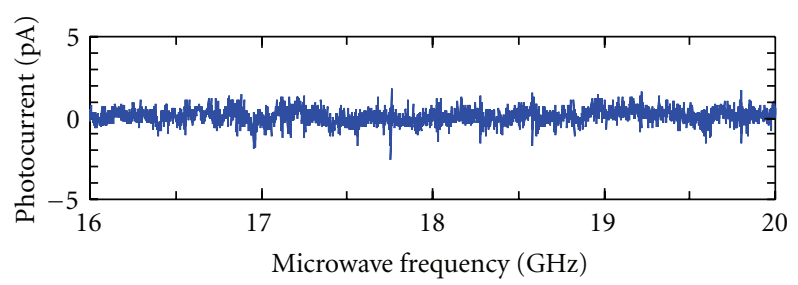

(b)

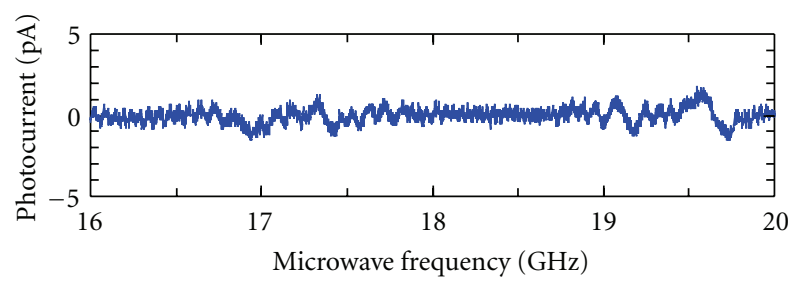

(c)

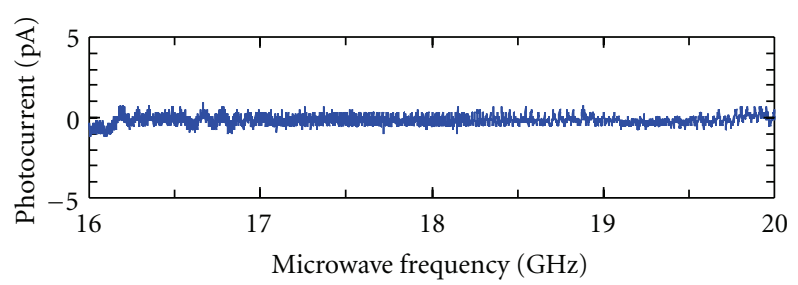

(d)

FIgURE 4: Dependence of the photocurrent on the microwave frequency for (a) dual-wavelength irradiation (optical beating at $1 \mathrm{THz}$ ), (b) single-wavelength irradiation (no optical beating), (c) no-irradiating while biasing the PC device, and (d) no-irradiating and no-biasing the PC device.

was applied to the PC device. The harmonics of the signal $(600 \mathrm{~Hz})$ created in the photocurrent were measured by a fast Fourier transformer (FFT).

Figure 6 shows $\eta$ (the ratio of each harmonic current to that of the fundamental wave) in the case of the opticalbeat frequency of 1, $2 \mathrm{THz}$, and single-wavelength irradiation of the laser light. The harmonic components due to the waveform distortion of the signal source itself are also shown (the triangles), which represents the lower sensitivity limit in our measuring system. The maximum order $(n=35)$ represents upper frequency limit in our measuring system. In the case of the optical beat of $1 \mathrm{THz}$ (the blue-colored circles), many harmonics were observed. The second-order harmonic rapidly decreases, and the harmonics over the third-order gradually decrease as the order of harmonics increases. In the case of the optical beat of $2 \mathrm{THz}$ (the yellowcolored circles), $\eta$ is slightly lower than that for the opticalbeat frequency of $1 \mathrm{THz}$. Therefore, $\eta$ tends to decrease as 


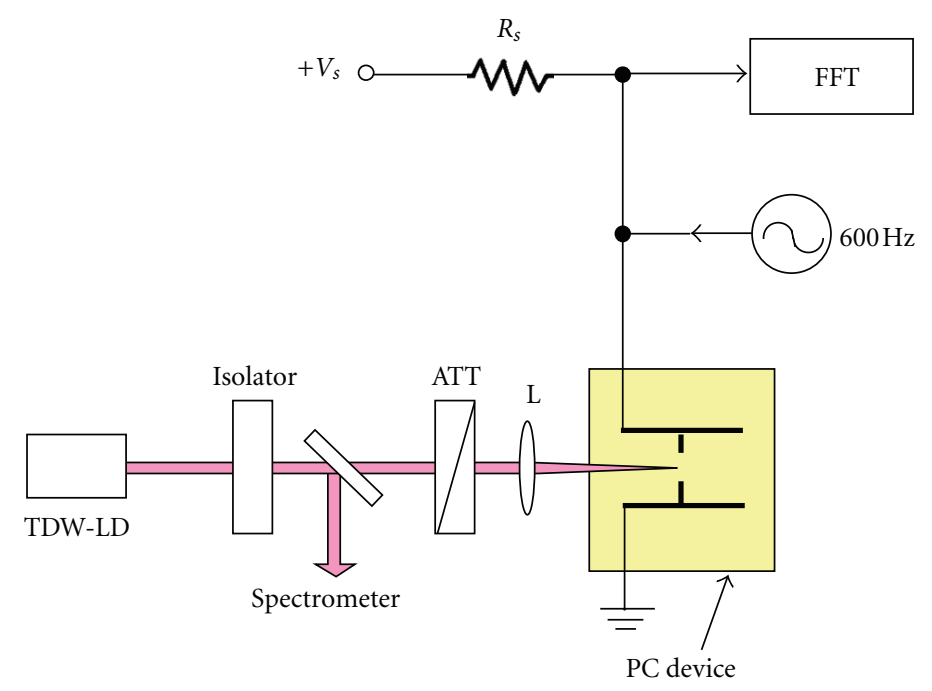

Figure 5: Experimental setup to measure the efficiency of the high-order harmonic generation in the PC device. The signal $(600 \mathrm{~Hz})$ was applied to the PC device, and the harmonics in the photocurrent were measured by a fast Fourier transformer (FFT).

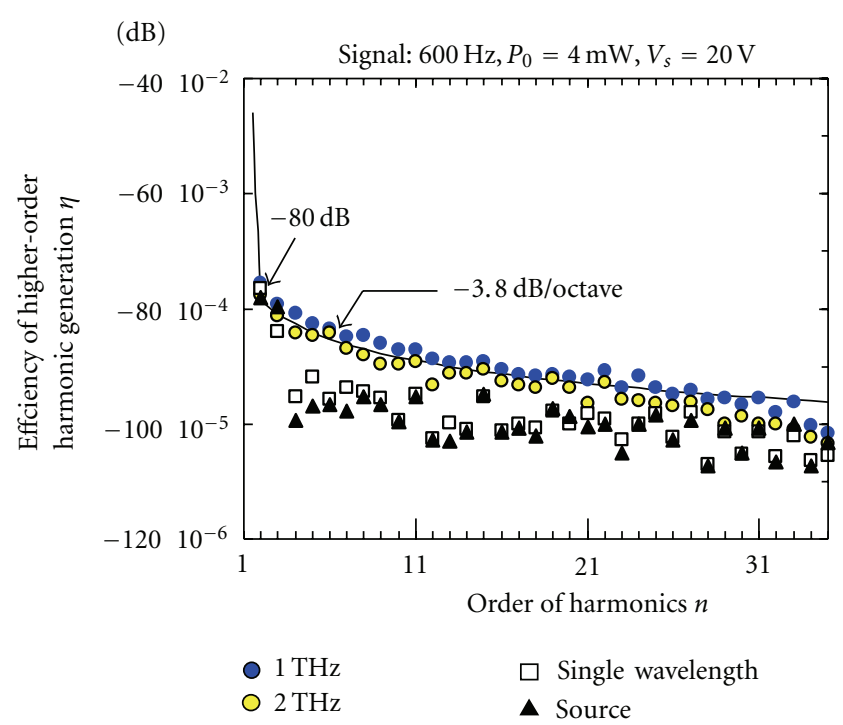

FIGURE 6: Efficiency of the high-order harmonic generation $(\eta)$ in the PC device (fundamental frequency: $600 \mathrm{~Hz}$ ). The source represents the lower sensitivity limit in the measuring system. $\eta$ in the cases of dual-wavelength irradiation (optical-beat frequency of 1 and $2 \mathrm{THz}$ ) is larger than that in the case of single-wavelength irradiation (no optical beating).

the optical-beat frequency increases. In the case of singlewavelength irradiation (i.e., no optical beating) of the laser light, the plots (the squares) almost overlap with those of the signal source (the triangles). Therefore, it is known that the harmonics in the case of the single-wavelength irradiation are below the lower sensitivity limit of the measuring system, or the harmonics are scarcely generated. These experimental results suggest that the current waveform of the signal $(600 \mathrm{~Hz})$ is distorted by the modulated photoconductance with the $\mathrm{THz}$ frequency, generating the high-order harmonics in the photocurrent. Detailed mechanism on the harmonic generation in the PC device is being investigated.
The solid line represents the fitted line to the plots for the optical beat of 1 and $2 \mathrm{THz} . \eta$ in $\mathrm{dB}$ is expressed as

$$
\eta= \begin{cases}-80 \mathrm{~dB} & (n=2), \\ -3.8 \mathrm{~dB} / \text { octave } & (n \geq 3),\end{cases}
$$

where the plots for $n \geq 32$ are not included in the fitting calculation because of low sensitivity at the band edge of the FFT. According to studies on the generation and homodyne detection of the $\mathrm{cw} \mathrm{THz}$ wave by the pioneers $[3,8]$, the PC device has an almost flat response up to $600 \mathrm{GHz}$ and a role-off response for over $600 \mathrm{GHz}$. The cutoff frequency $(600 \mathrm{GHz})$ depends on the time constant of the PC device tested. Therefore, when the low order harmonics of the microwave generated by the diode are, instead of the signal $(600 \mathrm{~Hz})$ mentioned above, applied to the PC device, the high-order harmonics with the sub- $\mathrm{THz}$ to $\mathrm{THz}$ region are generated in the PC device.

3.3. Measurement of the Optical-Beat Frequency. Using the periodic photocurrent peaks obtained when the microwave frequency was swept, the optical-beat frequency in the $\mathrm{THz}$ region was measured. The blue-colored peaks shown in Figure 7 are, the same plots as shown in Figure 4(a), the measured photocurrent variation for the optical-beat frequency of $1 \mathrm{THz}$ under the conditions of $P_{0}=4 \mathrm{~mW}$, $P_{\mathrm{mw}}=-40 \mathrm{dBm}$, and $V_{s}=20 \mathrm{~V}$. Figure 8 shows a schematic diagram of a double-stage harmonic generator consists of the diode and PC device. The $m$ th order harmonic is generated by the diode and fed to the PC device, and then the $n$th order harmonic is generated by the PC device from the $m$ th order harmonic fed from the diode. That is, the $m$ th order harmonic acts as the source of the $n$th order harmonic in the PC device. The harmonic frequency $f_{3}(i)$ defined by the orders $m$ and $n$ is expressed as

$$
f_{3}(i)=m n f_{c}(i) \quad(i=1,2, \ldots),
$$




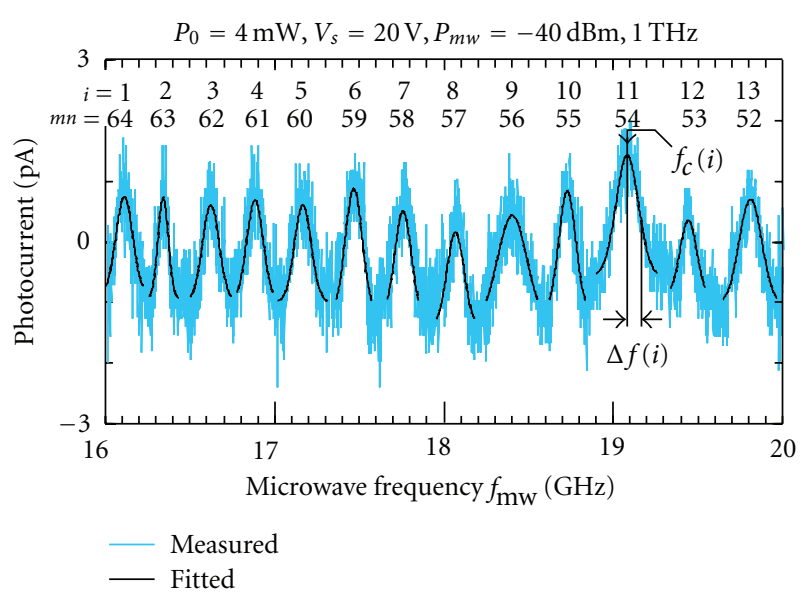

Figure 7: Determination of the central frequency $f_{c}(i)$ of the $i$ th peak for optical beating at $1 \mathrm{THz}$. The blue-colored plots (same as Figure 4(a)) represent the measured periodic peaks. The solid lines are the fitted curves defined by $(3)$ for each peak. $f_{c}(i)$ is determined as the frequency at each Gaussian top. $\Delta f(i)$ is the peak width for the $i$ th peak. Numerical examples for the seven peaks $(i=1-7)$ are listed in Table 1.

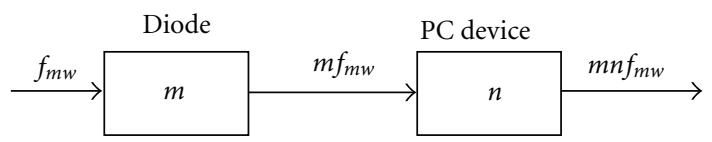

FIGURE 8: Double-stage harmonic generator consisting of the diode and PC device.

where $i$ is the identification number of the photocurrent peaks shown in Figure 7 and $f_{c}(i)$ is a central frequency of the microwave for the $i$ th peak. $f_{c}(i)$ is determined by fitting a following Gaussian function:

$$
y(i)=a_{i}+b_{i} \exp \left[-\left(\frac{f_{\mathrm{mw}}-f_{\mathrm{c}}(i)}{\sqrt{2} \Delta f(i)}\right)^{2}\right] \quad(i=1,2, \ldots),
$$

to the $i$ th peak, where $f_{\mathrm{mw}}$ is the swept frequency of the microwave, $a_{i}$ and $b_{i}$ are constants for each peak, and $\Delta f(i)$ is the peak width in which the photocurrent decreases to $1 / \sqrt{e}$ relative to that at the Gaussian top. The fitted curves are shown by the solid lines in Figure 7. Numerical examples of $f_{c}(i)$ and corresponding $m$ and $n$ for the seven peaks $(i=1$ to 7$)$ are listed in Table 1 .

The orders $m$ and $n$ are determined as follows. Since a maximum frequency of the signal fed to the PC device is considered to be restricted to $100 \mathrm{GHz}$ as described in Section 3.2, the order $m$ must satisfy

$$
m f_{\mathrm{c}}(i) \leq 100 \mathrm{GHz}
$$

In addition, using the frequency of $1028 \pm 5 \mathrm{GHz}$ measured by the homodyne detection described in Section 2 as the coarse frequency, the order $n$ must satisfy

$$
\left|f_{3}(i)-1028\right|<5 \mathrm{GHz}
$$

TABLe 1: Numerical examples of the central frequency $f_{c}(i)$ and its harmonic frequency $f_{3}(i)$ for the seven peaks in Figure 7 (opticalbeat frequency: $1 \mathrm{THz}$ ). $i$ is the identification number of the peak. $m$ and $n$ are the candidate orders of the harmonics for the diode and PC device, respectively. $m n$ is a total order of the harmonics. The asterisks are the determined combinations of $m$ and $n$ for each peak (see the text).

\begin{tabular}{lcccccc}
\hline$i$ & $f_{c}(i)(\mathrm{GHz})$ & $m$ & $n$ & $m n$ & $f_{3}(i)(\mathrm{GHz})$ & \\
\hline \multirow{2}{*}{1} & \multirow{2}{*}{16.10312} & 1 & 64 & 64 & 1030.600 & $*$ \\
& & 2 & 32 & 64 & 1030.600 & \\
\hline \multirow{2}{*}{1} & \multirow{2}{*}{16.34376} & 1 & 63 & 63 & 1029.657 & $*$ \\
& & 3 & 21 & 63 & 1029.657 & \\
\hline \multirow{3}{*}{3} & \multirow{2}{*}{16.61971} & 1 & 62 & 62 & 1030.422 & $*$ \\
& & 2 & 31 & 62 & 1030.422 & \\
\hline 4 & 16.88374 & 1 & 61 & 61 & 1029.908 & $*$ \\
\hline & & 1 & 60 & 60 & 1029.987 & $*$ \\
& & 2 & 30 & 60 & 1029.987 & \\
5 & \multirow{2}{*}{17.16645} & 3 & 20 & 60 & 1029.987 & \\
& & 4 & 15 & 60 & 1029.987 & \\
& & 5 & 12 & 60 & 1029.987 & \\
\hline 6 & 17.47100 & 1 & 59 & 59 & 1030.789 & $*$ \\
\hline \multirow{2}{*}{7} & \multirow{2}{*}{17.75825} & 1 & 58 & 58 & 1029.979 & $*$ \\
& & 2 & 29 & 58 & 1029.979 & \\
\hline
\end{tabular}

For the peak $i=1$ in Table 1 , three candidates of $(m=1$, $n=64),(2,32)$, and $(4,16)$ satisfy $(4)$ and (5). For the peak $i=2$, two candidates of $(1,63)$ and $(3,21)$ satisfy $(4)$ and (5). Whereas for the peak $i=4$ and $i=6$, only one candidate $(m=1)$ satisfies (4) and (5). As explained in Section 3.1, the periodic peaks were observed within the microwave power variation of about $\pm 3 \mathrm{~dB}$ around the optimum microwave power of $-40 \mathrm{dBm}$. That is, the periodic peaks are not generated when the microwave power is stronger or weaker than the optimum microwave power by over $3 \mathrm{~dB}$. While reasons for the dependence on the microwave power are not clarified at present, we utilize only its property to determine the harmonic order of the microwave. Since the efficiency of the harmonic generation of the diode is $-20 \mathrm{~dB} /$ octave, the power of the $2 \mathrm{nd}, 3 \mathrm{rd}, 4$ th, and 5 th harmonic generated by the diode is lower than that of the fundamental $(m=1)$ by $20,31.6,40$, and $46.4 \mathrm{~dB}$, respectively. Accordingly, the power differences among the harmonics for $m=1-5$ are much larger than $3 \mathrm{~dB}$. Hence, the order $m$ for all peaks must be common. Consequently, $m=1$ is determined to be the common order for all peaks. The resultant $n$ and $f_{3}(i)$ for $m=1$ are indicated by the asterisks in Table 1 . It is seen in Table 1 that the high-order harmonics with the frequencies up to the $\mathrm{THz}$ region are generated by the $\mathrm{PC}$ device from the fundamental $(m=1)$ of the microwave. The total orders $m n$ for each peak are shown in Figure 7. It can be understood that the periodic peak is generated when the harmonic with the 64th to 52th order is sequentially tuned to the optical beat as the microwave frequency is swept from 16 to $20 \mathrm{GHz}$. In addition, the high-order harmonics (the source of them is the $2 \mathrm{nd}, 3 \mathrm{rd}, 4 \mathrm{th}$, and 5 th order harmonic produced by the 


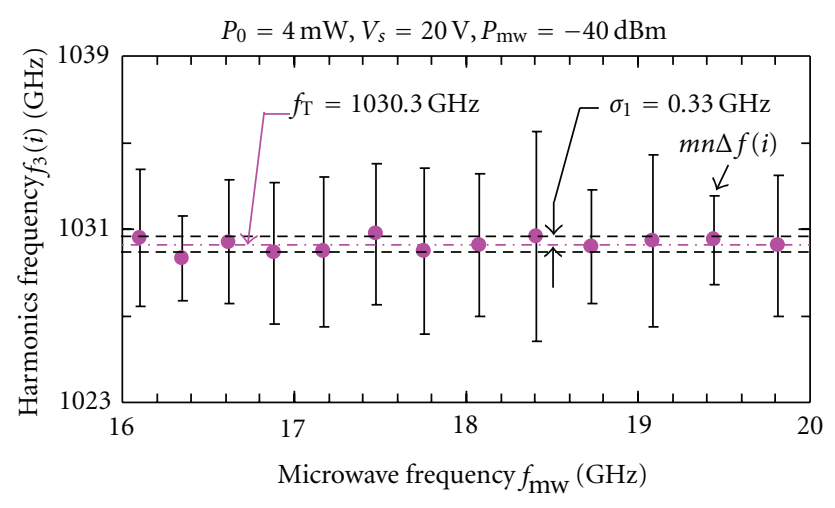

(a)

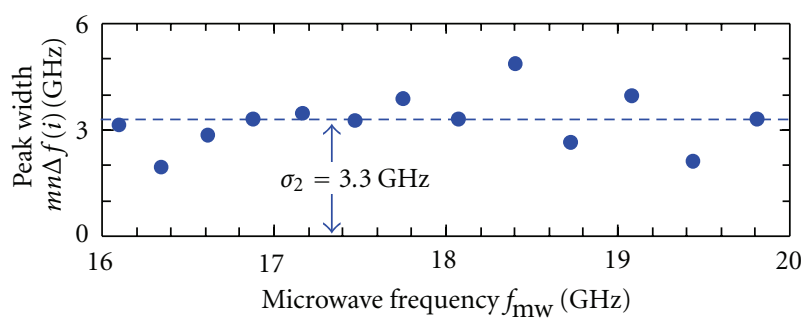

(b)

FIGURE 9: Distribution of (a) the determined harmonic frequency $f_{3}(i)$ (plots) and its peak width $m n \Delta f(i)$ (error bars) for the highorder harmonics, and (b) the peak width $m n \Delta f(i)$. The optical beat frequency $f_{\mathrm{T}}$ is defined as an average of $f_{3}(i) . \sigma_{1}$ and $\sigma_{2}$ are the standard deviations of $f_{3}(i)$ and $m n \Delta f(i)$, respectively. The random error is given by $\sigma_{1}+\sigma_{2}$ (see the text).

diode) are generated by the PC device. However, they does not contribute to the periodic peak generation, because the power levels for $m=2-5$ relative to that for $m=1$ are much lower than $3 \mathrm{~dB}$.

Distribution of the resultant frequency $f_{3}(i)$ is shown by the plots in Figure 9(a). The peak width $m n \Delta f(i)$ obtained by (3) is shown by the length of the upper and lower error bar and is also plotted in Figure 9(b). The optical-beat frequency $f_{\mathrm{T}}$ is defined as an average of $f_{3}(i)$ and given by

$$
f_{\mathrm{T}}=\frac{1}{\mathrm{M}} \sum_{i=1}^{\mathrm{M}} f_{3}(i)
$$

where $\mathrm{M}$ is the repeat times of the measurement. According to the ANSI/ASME performance test codes PTC19.1 [11], the total error $\delta_{\mathrm{T}}$ consists of the bias error $\delta_{\mathrm{B}}$ and random error $\delta_{\mathrm{R}}$ and is given by

$$
\delta_{\mathrm{T}}=\delta_{\mathrm{B}}+\delta_{\mathrm{R}}
$$

The bias error is constant and roughly estimated to be $\delta_{\mathrm{B}} \approx$ $0.1 \mathrm{GHz}$ (the frequency instability of the high-order harmonics of the microwave: $200 \mathrm{kHz}$ at $1 \mathrm{THz}$, and the data processing error: $50 \mathrm{MHz}$ ). The random error in our experiment is expressed as

$$
\delta_{\mathrm{R}}=\sigma_{1}+\sigma_{2},
$$

where $\sigma_{1}$ is the long-term frequency instability of the light source including the microwave generator, which is obtained as the standard deviation of the fluctuation of $f_{3}(i)$ shown in Figure 9(a) and given by

$$
\sigma_{1}=\sqrt{\frac{\sum_{i=1}^{\mathrm{M}}\left(f_{3}(i)-f_{\mathrm{T}}\right)^{2}}{\mathrm{M}-1}},
$$

$\sigma_{2}$ is the short-term frequency instability of the light source, and is obtained as the standard deviation for the $i$ th peak (namely, the peak width $m n \Delta f(i)) . \sigma_{2}$ is improved by Mtimes repetition measurements and given by

$$
\sigma_{2}=\sqrt{\frac{\sum_{i=1}^{\mathrm{M}}(m n \Delta f(i))^{2}}{\mathrm{M}}} .
$$

Substituting the measured frequencies $f_{3}(i)$ in Figure 9(a) to (6), the optical-beat frequency is determined to be $f_{\mathrm{T}}=1030.3 \mathrm{GHz}$, where $\mathrm{M}=13$, because the frequency measurements are repeated by 13 times during sweeping the microwave frequency from 16 to $20 \mathrm{GHz}$. By substituting $f_{\mathrm{T}}$ into (9), the long-term frequency instability is obtained to be $\sigma_{1}=0.33 \mathrm{GHz}$. Substituting $m n \Delta f(i)$ in Figure $9(\mathrm{~b})$ into (10), the short-term frequency instability is obtained to be $\sigma_{2}=3.3 \mathrm{GHz}$. Using $\sigma_{1}$ and $\sigma_{2}, \delta_{\mathrm{R}}=3.63 \mathrm{GHz}$ by (8). Finally, the total error results in $\delta_{\mathrm{T}}=3.73 \mathrm{GHz}$ by (7).

Since $\delta_{\mathrm{T}} \approx \sigma_{2}$, the total error is dominated by the frequency fluctuations of the light source (DW-DBR-LD). If the frequencies of the light source are completely stabilized (i.e., $\sigma_{2}=0$ ), the total error becomes $\delta_{\mathrm{T}}=\delta_{\mathrm{B}}+\sigma_{1}=$ $0.43 \mathrm{GHz}$. Since $\sigma_{1}$ includes the long-term frequency instabilities of the light source and can be reduced by improvements of the light source, the total-error limit (i.e., the measurement error) of the proposed method is estimated to be less than $0.43 \mathrm{GHz}$. $\delta_{\mathrm{T}}$ is reduced to about $1 / 12$ and $1 / 28$ compared with the frequency error of the transceiver-type homodyne detection $( \pm 5 \mathrm{GHz})$ and the optical spectrometer $( \pm 12 \mathrm{GHz})$, respectively. The total error of $0.43 \mathrm{GHz}$ is the same error as the Fourier-transformed interferometer with a scanning range of $0.35 \mathrm{~m}$.

The proposed method readily presents a compact frequency meter for the $\mathrm{cw} \mathrm{THz}$-wave generator with a photomixer. In addition, if the oscillation frequencies of the two laser beams are controlled so as to reduce the peak width of the photocurrent response, the frequency of the emitted $\mathrm{THz}$ wave can be stabilized, which will realize a highprecision signal generator in the $\mathrm{THz}$ region and a highresolution $\mathrm{THz}$ spectrometer.

\section{Conclusions}

A new method for measuring the frequency of the optical beat in the $\mathrm{THz}$ region is presented. By applying the microwave signal to the PC device via the step recovery diode, it has been found that the periodic peaks are caused in the photocurrent when the microwave frequency is swept. These peaks are generated by the homodyne detection of the optical beat with the high-order harmonics of the microwave when the frequency of the high-order harmonic coincides 
with the optical-beat frequency. Furthermore, it has been found that the high-order harmonics of the microwave are generated by the PC device due to the nonlinearity of the PC device and the efficiency of the high-order harmonic generation is about $-3.8 \mathrm{~dB} /$ octave. The physical mechanism of the harmonic generation by the PC device is being investigated.

The periodic peaks generated by the homodyne detection were observed when the microwave was swept from 16 to $20 \mathrm{GHz}$ with a power of $-40 \mathrm{dBm}$. Using the peak frequencies and the harmonic orders, the optical beat frequency was measured to be $1030.3 \mathrm{GHz}$. The frequency error was measured to be $3.73 \mathrm{GHz}$, which was dominated by the frequency fluctuation of the light source. The measurement error of the proposed method is estimated to be less than $0.43 \mathrm{GHz}$.

The proposed method is constructed by simply adding a diode and capacitor to a conventional PC device, realizing a compact $\mathrm{THz}$ frequency meter in a $\mathrm{cw} \mathrm{THz}$-wave generator with a photomixer. If the microwave signal generator is calibrated by a frequency standard, for example, a cesiumbased frequency standard, optical-beat frequencies in the $\mathrm{THz}$ region can be precisely measured.

\section{Acknowledgment}

The authors are grateful to Dr. Isao Morohashi of the National Institute of Information and Communications Technology (NICT) in Japan for helpful discussions on the PC device.

\section{References}

[1] P. Gu, M. Tani, M. Hyodo, K. Sakai, and T. Hidaka, "Generation of cw-terahertz radiation using a two-longitudinal-mode laser diode," Japanese Journal of Applied Physics, Part 2, vol. 37, no. 8B, pp. L976-L978, 1998.

[2] K. Kawase, K. Imai, and H. Ito, "Transform-limited, narrowlinewidth, terahertz-wave parametric generator," Applied Physics Letters, vol. 78, no. 19, pp. 2819-2821, 2001.

[3] E. R. Brown, K. A. McIntosh, K. B. Nichols, and C. L. Dennis, "Photomixing up to $3.8 \mathrm{THz}$ in low-temperaturegrown GaAs," Applied Physics Letters, vol. 66, no. 3, pp. 285287, 1995.

[4] N. C. Wong, "Optical frequency counting from UV to the near IR,” Optics Letters, vol. 17, no. 16, pp. 1155-1157, 1992.

[5] S. Matsuura, P. Chen, G. A. Blake, J. C. Pearson, and H. M. Pickett, "Two-frequency MOPA diode laser system for difference frequency generation of coherent THz-waves," in Terahertz Spectroscopy and Applications, vol. 3617 of Proceedings of the SPIE, pp. 14-21, January 1999.

[6] T. Yasui, Y. Kabetani, E. Saneyoshi, S. Yokoyama, and T. Araki, "Terahertz frequency comb by multifrequency-heterodyning photoconductive detection for high-accuracy, high-resolution terahertz spectroscopy," Applied Physics Letters, vol. 88, no. 24, Article ID 241104, pp. 1-3, 2006.

[7] S. Yokoyama, R. Nakamura, M. Nose, T. Araki, and T. Yasui, "Terahertz spectrum analyzer based on a terahertz frequency comb," Optics Express, vol. 16, no. 17, pp. 13052-13061, 2008.

[8] S. Verghese, K. A. McIntosh, S. Calawa, W. F. Dinatale, E. K. Duerr, and K. A. Molvar, "Generation and detection of coherent terahertz waves using two photomixers," Applied Physics Letters, vol. 73, no. 26, pp. 3824-3826, 1998.

[9] S. Iio, M. Suehiro, T. Hirata, and T. Hidaka, "Two-longitudinal-mode laser diodes," IEEE Photonics Technology Letters, vol. 7, no. 9, pp. 959-961, 1995.

[10] K. Murasawa and T. Hidaka, "Electrically tunable and dualwavelength semiconductor laser with a liquid crystal display," Japanese Journal of Applied Physics, vol. 48, no. 2, Article ID 022202, pp. 1-5, 2009.

[11] American National Standards Institute, "Measurement uncertainty," in Supplement on Instruments and Apparatus, Part 1, ANSI/ASME performance test codes PTC19.1-1985, American Society of Mechanical Engineers, New York, NY, USA, 1985. 

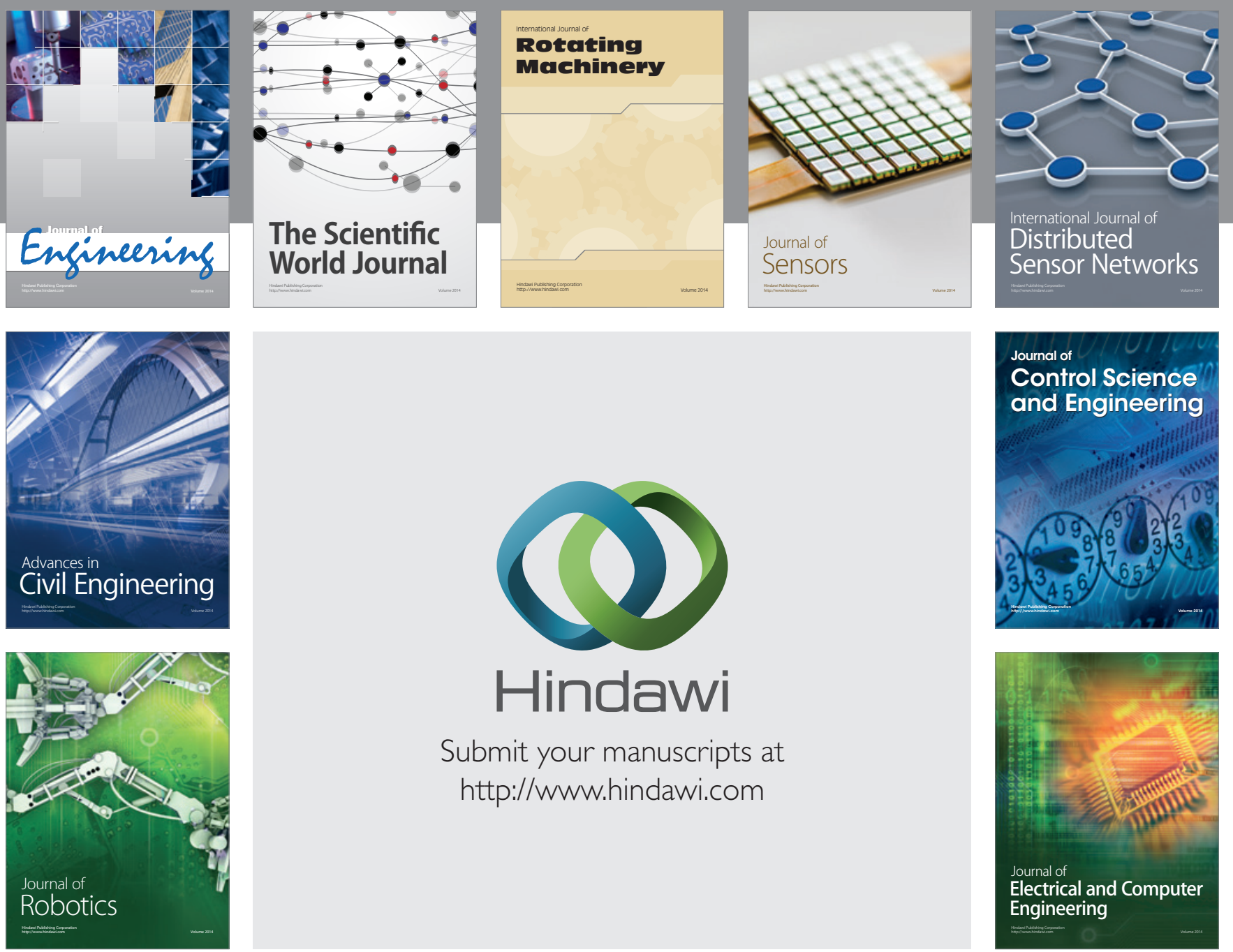

Submit your manuscripts at

http://www.hindawi.com
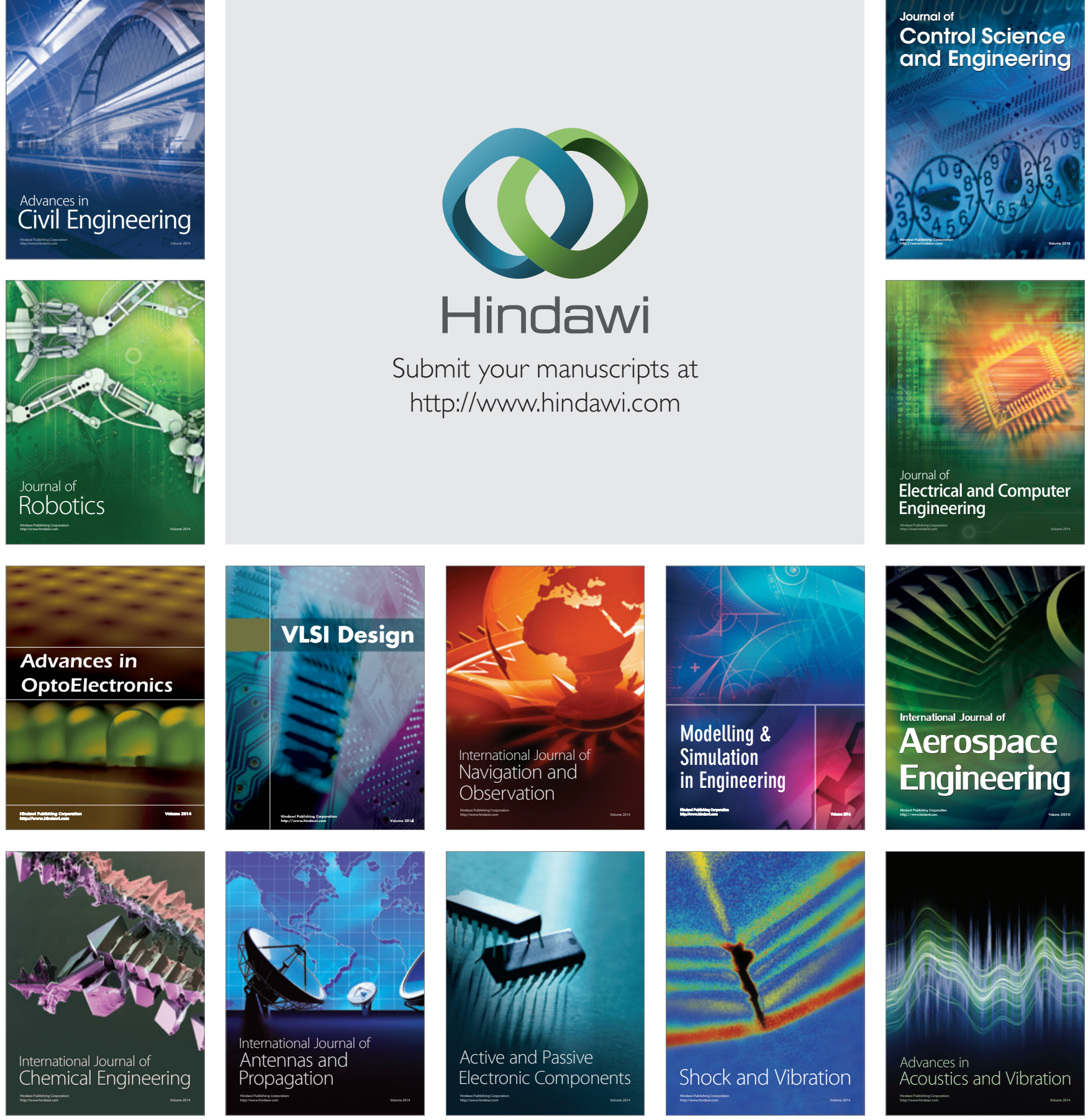